\title{
Single Molecule and Cell Detection Devices for Diagnostics and Therapeutics in Precision Medicine
}

\author{
Daisuke Onoshima \\ Institute of Innovation for Future Society, Nagoya University \\ Nagoya, Japan
}

According to the Precision Medicine Initiative, precision medicine is "an emerging approach for disease treatment and prevention that takes into account individual variability in genes, environment, and lifestyle for each person. To ensure the safety and maximum therapeutic effects, our group have developed in vivo and in vitro detection techniques with bioimaging devices for the analysis of behavior, accumulation, and condition of single DNA molecules and stem cells.

Fluorescent imaging is expected to detect the transplanted cells with higher sensitivity in comparison to other imaging modalities. In vivo fluorescence imaging techniques, which can detect and analyze the fluorescence or emission from the body, have been developed for small animals such as mice and rats. Quantum dots (QDs) can be utilized in these devices as bio-probes or labels for biological imaging of cells and tissues. They have developed into new formats of biosensing to push the limits of detection. The potential clinical applications of QDs have been expanded by the development of considerably low cytotoxicity QDs that do not include cadmium or selenium, as well as the development of longwave fluorescence QDs with strong permeability into the body. In vitro imaging techniques have been developed based on the microfabricated devices to control or manipulate single cells constrained in a small space. These devices are primed to be powerful tools to provide the basis for the detection of small amounts of samples and simple operation. They have been also known to facilitate single-molecule measurements of DNA methylation, especially in combination with high-resolution microscopy. Such approaches are useful to determine tumorigenic potentials of cells that may otherwise be masked by ensemble averages. 